\title{
Energy loss and modification of photon-tagged jets with ATLAS
}

\author{
Dennis V. Perepelitsa*, on behalf of the ATLAS Collaboration \\ University of Colorado Boulder \\ E-mail: dvpecern.ch
}

\begin{abstract}
Events containing a high-transverse momentum $\left(p_{\mathrm{T}}\right)$ prompt photon offer a useful tool to study the dynamics of the hot, dense medium produced in heavy ion collisions. Because photons do not carry color charge, they are unaffected by the medium, and thus provide information about the momentum, direction, and flavor (quark or gluon) of the associated hard-scattered parton before it begins to shower and becomes quenched. In particular, the presence of a high- $p_{\mathrm{T}}$ photon can be used to select $p p$ and $\mathrm{Pb}+\mathrm{Pb}$ events with the same configuration before quenching, limiting the effects of selection biases present in other jet measurements. This proceedings reports results from the ATLAS detector using the large statistics $p p$ and $\mathrm{Pb}+\mathrm{Pb}$ data delivered by the LHC in 2015 , including measurements of the overall parton energy loss (via the photon+jet $p_{\mathrm{T}}$ balance) and the modification of the component of the shower which remains correlated with the initial parton direction (via the longitudinal fragmentation function in cone). Since all results are fully corrected for detector effects with unfolding procedures, direct, systematic comparisons to state of the art theoretical models can be made.
\end{abstract}

International Conference on Hard and Electromagnetic Probes of High-Energy Nuclear Collisions 30 September - 5 October 2018

Aix-Les-Bains, Savoie, France

* Speaker. 


\section{Introduction}

Photon-tagged measurements of jet energy loss and modification exploit the fact that the outgoing photon is unmodified by its passage through the hot nuclear medium created in $\mathrm{Pb}+\mathrm{Pb}$ collisions. The large luminosities of $\mathrm{Pb}+\mathrm{Pb}$ and $p p$ collision data delivered by the LHC in 2015 has enabled a series of high-statistics measurements in these events. These include measurements sensitive to the absolute amount of energy lost outside of a fixed jet cone radius, to how jet structure and substructure are modified, and to where the lost energy or the medium response is distributed in angular and momentum space.

This proceedings present ATLAS measurements of the photon-jet $p_{\mathrm{T}}$ balance [1] and the photon-tagged jet fragmentation function [2] in $p p$ and $\mathrm{Pb}+\mathrm{Pb}$ collisions. A particular focus is the correction for finite resolution effects on kinematic quantities, resulting in distributions reported at the final-state particle level. Because of this improvement, the data can be compared directly for the first time to other data, the predictions of event generators, or models.

\section{ATLAS detector and event reconstruction}

The measurements summarized here utilize multiple aspects of the ATLAS detector, including the full electromagnetic and hadronic calorimeter systems, the inner detector, and the high-level trigger system. A detailed description of the detector can be found in Ref. [3]. For both measurements, $p p$ and $\mathrm{Pb}+\mathrm{Pb}$ events are selected for analysis via the presence of a high- $p_{\mathrm{T}}$ photon in the high-level trigger, which is unprescaled and fully efficient for the kinematic range presented here. The centrality of events is characterized by the sum of the transverse energy in both forward calorimeter modules, situated at pseudorapidity $|\eta|=3.1-4.9$.

Jets are reconstructed by applying the anti- $k_{\mathrm{T}}$ algorithm with $R=0.4$ to energy deposits arranged into $\Delta \eta \times \Delta \varphi=0.1 \times 0.1$ logical towers in the calorimeter system. The jet reconstruction procedure features an iterative estimation and subtraction of the underlying event (UE) contribution to each tower. This estimate is performed event-by-event, in a pseudorapidity- and layer-dependent manner, and includes the effects of UE modulation from flow. The measured jet kinematics are calibrated using multiplicative factors based on simulation and on data-to-simulation comparisons of the in situ photon/Z+jet balance in $p p$ collisions. More information on jet reconstruction and performance in Run 2 heavy ion data may be found in Ref. [4].

Photon candidates are formed from calorimeter clusters according to the procedure used in proton-proton collisions, but operating on calorimeter cells after UE subtraction. They are required to pass a series of selection cuts on calorimetric shower shapes. Photons are furthermore required to be experimentally isolated via the sum of the transverse energy in calorimeter towers, evaluated after UE subtraction, within a cone of $R=0.3$ around the photon axis. This value, $E_{\mathrm{T}}^{\text {iso }}$, is required to be smaller than $3 \mathrm{GeV}(10 \mathrm{GeV})$ in $p p(\mathrm{~Pb}+\mathrm{Pb})$ collisions. More information on the photon reconstruction and performance in heavy ion collisions may be found in Ref. [5].

Charged-particle tracks are reconstructed in the inner detector systems, with selection criteria optimized for the high-occupancy heavy ion environment. More details are provided in Ref. [6].

In both analyses summarized below, events are selected for analysis via the presence of identified, isolated photons in the region $|\eta|<1.37$ or $1.56<|\eta|<2.37$. For the $p_{\mathrm{T}}$-balance analysis, 

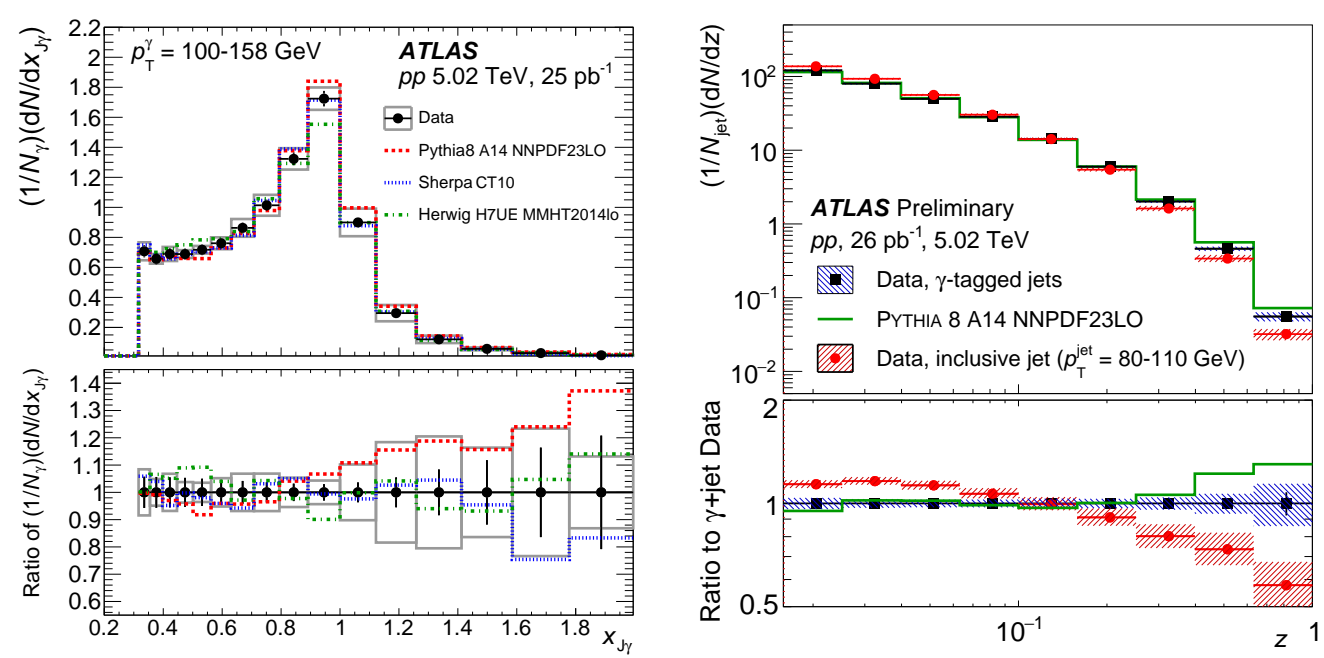

Figure 1: Measurements in $p p$ collisions of the photon-jet $p_{\mathrm{T}}$ balance (left, from Ref. [1]) and photontagged jet FF (right, from Ref. [2]), compared with the analogous distributions in event generators.

all jets within $|\eta|<2.8$ are considered, whereas for the fragmentation function (FF) analysis jets are required to lie within $|\eta|<2.1$ so that charged particles in the jet cone are contained within the tracking acceptance.

\section{Photon-jet transverse momentum balance}

High- $p_{\mathrm{T}}$ photons are paired inclusively with all jets satisfying $p_{\mathrm{T}}^{\text {jet }}>31.6 \mathrm{GeV}$ and an azimuthal separation $\Delta \varphi^{\gamma+\text { jet }}>7 \pi / 8$, and the jet-to-photon $p_{\mathrm{T}}$ ratio, $x_{\mathrm{J} \gamma}$, is recorded. In $\mathrm{Pb}+\mathrm{Pb}$ collisions, the distributions are first corrected for the presence of combinatoric photon-jet pairs by evaluating the per-photon rate of these pairs in simulated photon-jet events overlaid with minimum bias $\mathrm{Pb}+\mathrm{Pb}$ data events. In both $p p$ and $\mathrm{Pb}+\mathrm{Pb}$ events, these distributions are further corrected for the finite purity of the photon selection (which results in an admixture of hadron fragments, typically $\pi^{0}$ decay photons) via a double sideband technique.

The background-subtracted $x_{\mathrm{J} \gamma}$ yields are corrected for bin migration and finite efficiency effects via a two-dimensional unfolding procedure similar to that previously used for dijet events in Ref. [7]. After all corrections, $x_{\mathrm{J} \gamma}$ distributions are reported in differential selections on the photon $p_{\mathrm{T}}$ and event centrality.

The left panel of Fig. 1 shows the $\left(1 / N_{\gamma}\right)\left(\mathrm{d} N / \mathrm{d} x_{\mathrm{J} \gamma}\right)$ distribution for one photon $p_{\mathrm{T}}$ selection in $p p$ collisions, compared to generator-level predictions from Pythia, Sherpa and Herwig. Correcting for resolution effects recovers a sharp peak in the measured $x_{\mathrm{J} \gamma}$ distributions, also observed in event generators, indicative of a strong $p_{\mathrm{T}}$ correlation between photon-jet pairs in $p p$ collisions.

Figure 2 reports the $x_{\mathrm{J} \gamma}$ distribution in $\mathrm{Pb}+\mathrm{Pb}$ events of all centralities for the same photon $p_{\mathrm{T}}$ selection. In peripheral events, the $x_{\mathrm{J} \gamma}$ distribution is compatible with that in $p p$ collisions within uncertainties, indicating that energy loss effects are small. In progressively more central events, the $x_{\mathrm{J} \gamma}$ distribution is systematically deformed: the mean is shifted to the left, and the yield at small (large) $x_{\mathrm{J} \gamma}$ is increased (decreased). Notably, a $p p$-like peak at $x_{\mathrm{J} \gamma} \approx 0.9$ remains even in central 


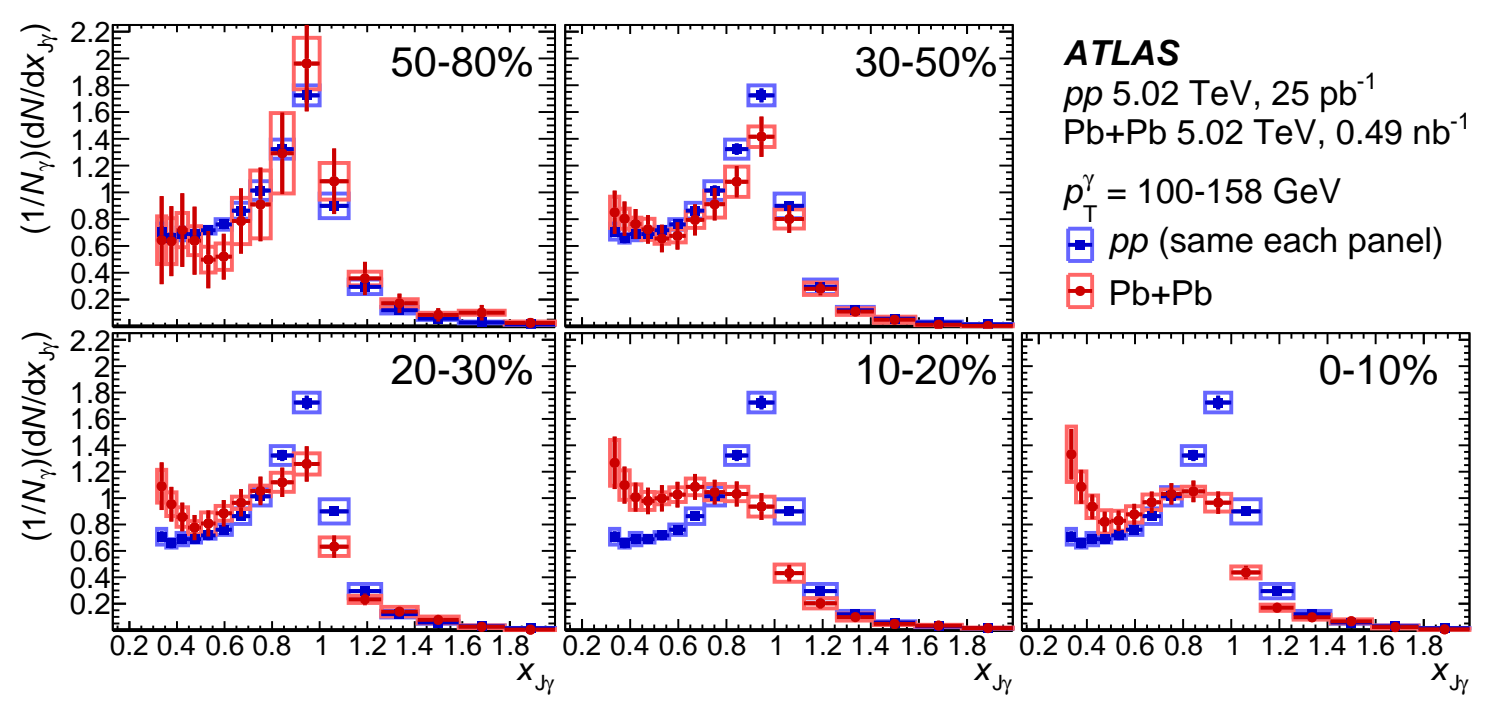

Figure 2: Comparison of the per-photon jet yield as a function of $x_{\mathrm{J} \gamma}$ in $\mathrm{Pb}+\mathrm{Pb}$ (red, each panel a different centrality selection) and $p p$ collisions (blue), from Ref. [1].
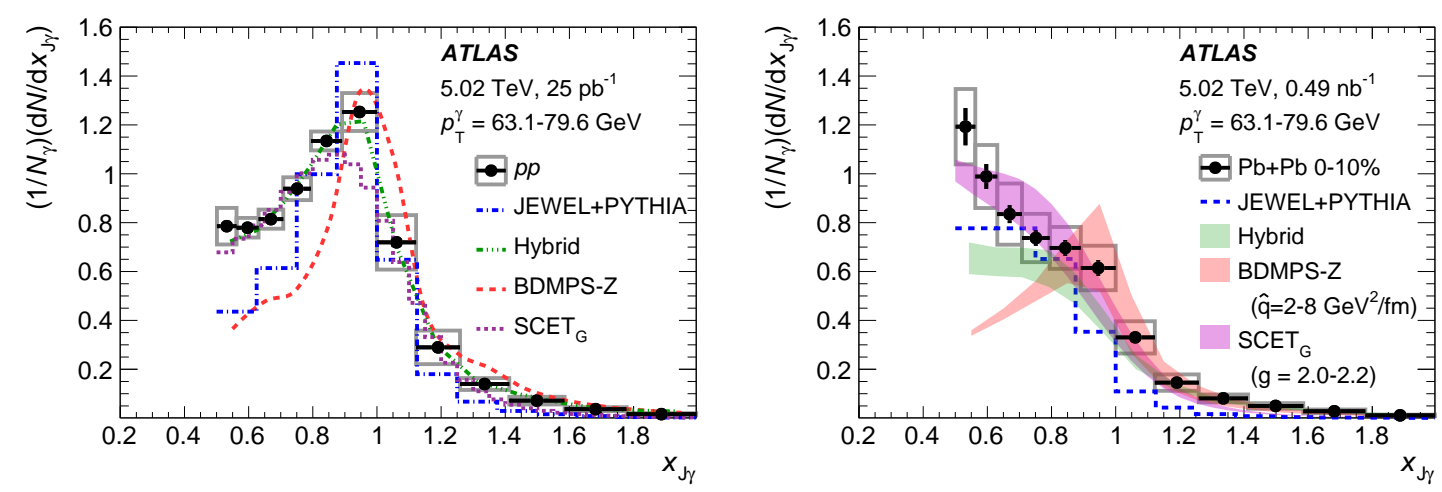

Figure 3: Example comparisons of fully unfolded $x_{\mathrm{J} \gamma}$ results in $p p$ collisions (left) and $\mathrm{Pb}+\mathrm{Pb}$ collisions (right) to theoretical calculations. See Ref. [1] for further details and references to the theoretical calculations shown here.

collision. This suggests the presence of jets which have lost minimal or no energy and thus remain well-correlated in $p_{\mathrm{T}}$ with the photon.

In Figure 3 the data are compared to a suite of energy loss MC models and calculations.

\section{Photon-tagged jet fragmentation function}

The internal structure of jets with $p_{\mathrm{T}}=63.1-144 \mathrm{GeV}$ in events with a $p_{\mathrm{T}}=79.6-126 \mathrm{GeV}$ photon are analyzed. The particular choice of kinematics is used to ensure that only the leading jet opposite to the photon, which is likely to be quark-initiated, enters into the analysis. Corrections are applied for three sources of backgrounds: (1) jets from combinatoric photon-jet pairs entering into the analysis, (2) UE charged particles falling into the jet cone, and (3) hadron fragments recon- 

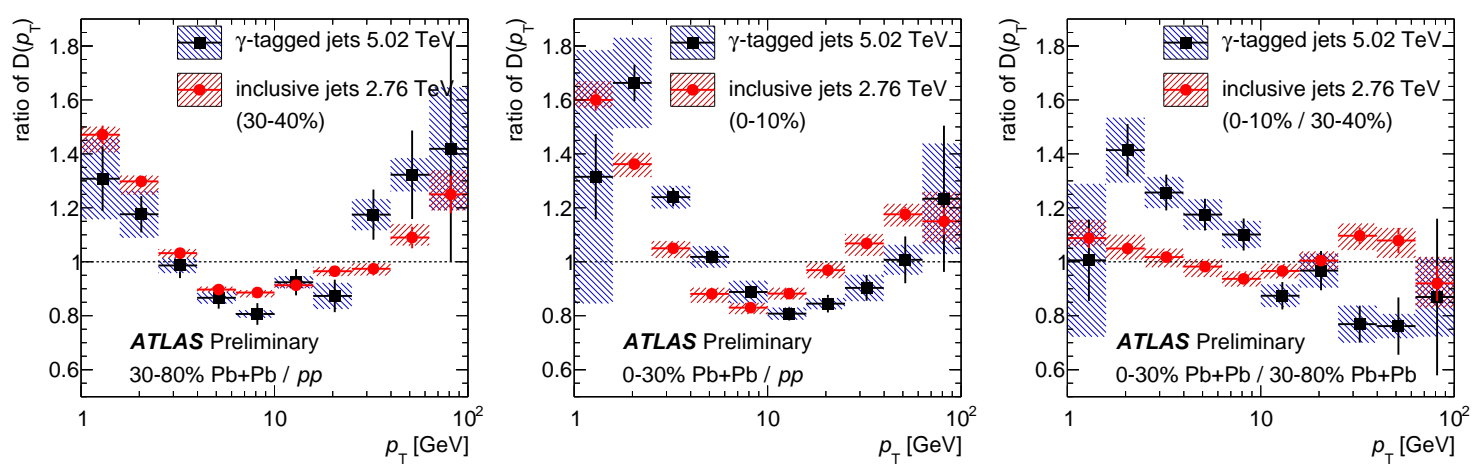

Figure 4: Ratios of the jet $\mathrm{FF}$ as a function of hadron $p_{\mathrm{T}}$ between different event types, comparing photontagged jets (black) to inclusively-selected jets (red). Figures from Ref. [2].

structed as photons. These backgrounds are constrained with data-driven methods and subtracted statistically. Bin migration effects are corrected with a two-dimensional unfolding procedure similar to that used in previous FF measurements [6].

The FF for photon-tagged jets in $p p$ collisions is presented as a function of $z=p_{\mathrm{T}}^{\text {hadron }} / p_{\mathrm{T}}^{\text {jet }}$ in the right panel of Fig. 1. It is systematically harder than the FF for inclusively-selected jets in a similar $p_{\mathrm{T}}$ range, consistent with the expectation from the higher quark-initiated jet fraction.

Figure 4 shows the ratio of the $\mathrm{FF}$ between $\mathrm{Pb}+\mathrm{Pb}$ and $p p$ collisions. In $30-80 \%$ events, the FF is modified in a way quantitatively similar to that of inclusive jets. However, in $0-30 \%$ central events, the modification is qualitatively different, with additional enhancement (suppression) at low (high) hadron $p_{\mathrm{T}}$. This stronger centrality dependence is compared to that for inclusive jets in the right panel of Fig. 4.

\section{Conclusion}

This proceedings briefly summarizes measurements by ATLAS of how photon-tagged jets lose energy and are modified through interactions with the hot nuclear medium created in $\mathrm{Pb}+\mathrm{Pb}$ collisions. Further information, data, and model comparisons may be found in Refs. [1, 2].

\section{References}

[1] ATLAS Collaboration, arXiv:1809.07280

[2] ATLAS Collaboration, ATLAS-CONF-2017-074, https://cds.cern.ch/record/2285812

[3] ATLAS Collaboration, JINST 3 (2008) S08003

[4] ATLAS Collaboration, arXiv:1805.05635

[5] ATLAS Collaboration, Phys. Rev. C93 (2016) 034914

[6] ATLAS Collaboration, Phys. Rev. C98 (2018) 024908

[7] ATLAS Collaboration, Phys. Lett. B774 (2017) 379 